\title{
Family Home Caregivers for Old Persons in the Arab Region: Perceived Challenges and Policy Implications
}

\author{
Azza 0. Abdelmoneium, Sanaa T. Alharahsheh \\ Doha International Family Institute, Doha, Qatar \\ Email: aabdelmoneium@qf.org.qa, salharahsheh@qf.org.qa \\ Received 10 December 2015; accepted 25 January 2016; published 29 January 2016 \\ Copyright (C) 2016 by authors and Scientific Research Publishing Inc. \\ This work is licensed under the Creative Commons Attribution International License (CC BY). \\ http://creativecommons.org/licenses/by/4.0/

(c) (i) Open Access

\begin{abstract}
The profound demographic changes that the Arab region has experienced during the past few decades have led to slow, albeit noteworthy increase in the proportion of older people, which will inevitably place heavy burdens on families who are assumed the responsibility in providing care for its older members. Although there has been much research about elderly caregiving issues in other regions around the world, less is known about aging family member caregiving, the challenges that family member caregivers face in the Arab region, and the facilities provided for family support to the elderly and to the caregivers themselves. Thus, the purposes of this study are to gain an overview of the current situation of ageing and elderly family member caregiving in the Arab region; to address the challenges family caregivers face, such as necessary skills and knowledge, communication, cultural issues, health, financial burdens and enhancement of quality of life in association with their care-giving role. These challenges can negatively impact the quality of life for the caregiver and the care receiver. The importance of implementing research and policy intervention to meet these challenges for all concerned will be discussed.
\end{abstract}

\section{Keywords}

Family, Caregiver, Old Person, Aging, Arab Region

\section{Introduction}

During the past few decades the Arab region has witnessed profound demographic changes that have led to a remarkable modification in the age structure of their population and a gradual increase in the proportion of older adults. The Arab region has been experiencing what Palloni and colleagues called a "silent aging process" [1], 
which is an outcome of rapid and concentrated declines in fertility and infant mortality, as well as a continuing increase in life expectancy. The percentage of older persons (65 years and above) in the Arab region increased from $3.6 \%$ in 1980 to $4.1 \%$ in 2010 , and is expected to reach $11.7 \%$ by 2050 [2]. This slow, albeit considerable, increase in the proportion of older people is becoming a new concern in the region as a whole [3].

Research carried out in the Arab world revealed that with the growing number and percentage of older adults in the region, chronic, non-communicable diseases and disabilities among the older Arab population would increase rapidly. Such trends are already placing heavy burdens on families who are assumed to be the main care provider for their older members [4] [5]. In some cases, however, families are simply not equipped or have the necessary skills and knowledge to meet the needs of their elderly relatives, especially those with a sever disability: one result has been the emergence of the formal care (institutionalized care) [6] [7].

Social science researchers have identified two types of home caregivers for older persons and categorized by scholars in studies as professional care and personal care. Professional care is skilled care provided by a nurse, physical therapist, occupational therapist, speech therapist or social worker. Personal care is provided by family members or workers at home, which is structurally based on gender relations whereby women take the responsibility for home care. In this paper we will look at personal care and especially family caregivers [8].

In the Arab world, the family, as a social institution, is the main source of support and care for its older members, helping them with day-to-day activities and giving them financial assistance and emotional support (Abyad, 2001) [9]. However, with the increase in women's participation in the work force, industrialization and a decline in the extended family, family caregivers are more likely to face physical, psychological, cultural and financial challenges associated with their care-giving role, which can impact negatively on the quality of life of those responsible for care and overall well-being [10] (Sinunu et al., 2009; Yount et al., 2009).

Research has shown that people recover faster when surrounded by their loved ones or in the comfort of their homes. Most people tend to find hospitals and other institutions quite traumatizing, which hampers their recovery efforts. Taking care of loved ones at home helps them to maintain their dignity and independence. A majority of elderly people prefer to spend their final years in their home where they can have as much freedom as they desire [11]. Although there have been many studies and much discussions about home care-giving issues in other regions around the world, the challenges that the family caregivers face in the Arab region are not well addressed in the literature dealing with the region.

The objective of this paper therefore is to describe and address the challenges that families face in taking care of their older members in the Arab region without reference to a specific group of older persons with specific needs. It will also address the research gap and policy implication for intervention. Key issues addressed include: 1) general trends in the demography of aging in Arab countries, 2) an overview of family care-giving for older persons in the Arab region, 3) challenges facing family caregivers, and 4) research and policy implications, and finally 5) a conclusion.

\section{Aging in the Arab World-An Emerging Phenomenon}

The process of aging in the Arab region is a relatively new phenomenon, even though it is found almost everywhere in the world. The aging population is the outcome of the transition from high to low fertility levels and the continuing increase in life expectancy [12] (Yount et al., 2009; ESCWA 2008; ESCWA, 2014). During the past few decades the fertility level in the Arab Region has decreased from 6.3 children per woman in 1980-1985 to 3.2 in 2010-2015, and expected to drop to 2.01 by 2050. While fertility decline is likely in all Arab countries, the rate of decline is projected to vary largely between countries of the region. Some countries, like Lebanon, Tunisia, United Arab Emirates, Bahrain and Qatar are below or at, the replacement level (2.1 children per woman), while other countries such as Sudan, Iraq, Palestine, Yemen, Comoros, Mauritania and Somalia have total fertility rates above 4 children per woman. Somalia has the highest fertility rate in the Arab Region (6.6 children per woman) (see Table 1) [13] [14].

Similarly, in the past three decades a substantial decline in Infant Mortality Rate (IMR) has been achieved in most of Arab countries, but again this rate varies between countries in the region. The average IMR in the Arab region dropped considerably from 64.8 per 1000 live births in the period 1980-1985 to 28.3 per 1000 in the period in 2010-2015, and is expected to drop to 14.8 per 1000 live births in the period 2045-2050. The greatest decline in infant mortality has occurred in Egypt (from 106.9 infant deaths per 1000 live births to 18.9 deaths per 1000 live births), followed by Yemen (from 117.0 to 56.2 per 1000), Morocco (from 83.9 to 26.3 per 1000), 
Table 1. Total fertility rate in the world and Arab countries 1980-2050.

\begin{tabular}{|c|c|c|c|c|}
\hline Country/area & 1980-1985 & 2010-2015 & 2025-2030 & 2045-2050 \\
\hline World & 3.60 & 2.50 & 2.37 & 2.24 \\
\hline Algeria & 6.49 & 2.82 & 2.20 & 1.93 \\
\hline Morocco & 5.40 & 2.78 & 2.27 & 1.96 \\
\hline Tunisia & 4.92 & 2.02 & 1.79 & 1.76 \\
\hline Egypt & 5.20 & 2.79 & 2.37 & 2.03 \\
\hline Libya & 7.34 & 2.38 & 1.82 & 1.62 \\
\hline Sudan & 6.63 & 4.46 & 3.55 & 2.81 \\
\hline Jordan & 7.05 & 3.27 & 2.56 & 2.05 \\
\hline Lebanon & 3.75 & 1.51 & 1.50 & 1.63 \\
\hline Palestine & 7.05 & 4.05 & 3.21 & 2.57 \\
\hline Iraq & 6.35 & 4.06 & 3.31 & 2.69 \\
\hline Syria & 6.77 & 3.00 & 2.37 & 1.92 \\
\hline Bahrain & 4.63 & 2.10 & 1.81 & 1.69 \\
\hline Kuwait & 5.03 & 2.60 & 2.36 & 2.18 \\
\hline Qatar & 5.45 & 2.05 & 1.75 & 1.67 \\
\hline Saudi Arabia & 7.02 & 2.68 & 2.10 & 1.78 \\
\hline U.A.E & 5.23 & 1.82 & 1.62 & 1.64 \\
\hline Oman & 8.32 & 2.91 & 2.08 & 1.71 \\
\hline Yemen & 9.23 & 4.15 & 2.82 & 2.03 \\
\hline Comoros & 7.05 & 4.74 & 3.90 & 3.13 \\
\hline Djibouti & 6.26 & 3.42 & 2.67 & 2.17 \\
\hline Mauritania & 6.28 & 4.70 & 3.84 & 3.05 \\
\hline Somalia & 7.07 & 6.61 & 5.13 & 3.63 \\
\hline
\end{tabular}

Source: 1) United Nation, Department of Economic and Social Affairs, World Population Prospects: The 2012 Revision, http://esa.un.org/unpd/wpp/unpp/panel_indicators.htm (The Medium Variant).

Oman (from 59.4 to 7.3 per 1000), Tunisia (from 65.8 to 15.5 per 1000), and Algeria (from 75.4 to 26.4 per 1000).

While the lowest IMRs are found in the GCC countries (United Arab Emirates with 5.7 per 1000; Qatar with 6.5 per 1000; Bahrain with 6.9 per 1000; Oman with 7.3 per 1000; Kuwait with 8.6 per 1000; and Saudi Arabia with 11.2 per 1000) and Lebanon (8.3 per 1000) which are lower than the world average of 36.8 per 1000 live births for the period 2010-2015, countries such as Somalia (79.5 per 1000), Mauritania (71.7 per 1000), Comoros (67.2 per 1000), Yemen (56.2 per 1000), Djibouti (55.3 per 1000) and the Sudan (55.0 per 1000) are registered as having higher IMRs than the world average for the same period 2010-2015 (United Nations 2012; ESCWA 2013) (see Table 2).

In addition, notable and continuing increases in life expectancy at birth for both males and females were achieved in the Arab region during the past three decades. The average life expectancy at birth for the region increased from 62 years in 1980-1985 to 70.7 years in 2010-2015, and is projected to increase further, reaching 76.8 years in 2045-2050. Currently the majority of Arab countries have life expectancies between 70 and 79 years, with the exception of Iraq (69.4 years), Yemen (63.0 years), the Sudan (61.9 years), Djibouti (61.6 years), Mauritania (61.5 years), Comoros (60.8 years), and Somalia (54.9 years) (see Figure 1) (Ibid).

As a result of the rapid declines in infant mortality, increases in life expectancy and decades of high fertility 
Table 2. Infant mortality rates in the world and Arab countries 1980-2050 (Per 1000 live births).

\begin{tabular}{|c|c|c|c|c|}
\hline Country/area & 1980-1985 & 2010-2015 & $2025-2030$ & $2045-2050$ \\
\hline World & 70.9 & 36.8 & 27.4 & 18.3 \\
\hline Algeria & 75.4 & 26.4 & 19.6 & 14.3 \\
\hline Bahrain & 23.1 & 6.9 & 4.8 & 3.3 \\
\hline Comoros & 105.6 & 67.2 & 54.9 & 42.7 \\
\hline Djibouti & 87.4 & 55.3 & 38.7 & 25.5 \\
\hline Egypt & 106.9 & 18.9 & 11.4 & 7.3 \\
\hline Iraq & 43.3 & 28.1 & 19.1 & 12.3 \\
\hline Jordan & 42.7 & 17.1 & 11.0 & 6.8 \\
\hline Kuwait & 23.9 & 8.6 & 6.1 & 4.2 \\
\hline Lebanon & 35.0 & 8.3 & 5.5 & 4.0 \\
\hline Libya & 51.0 & 13.8 & 8.3 & 5.9 \\
\hline Mauritania & 89.2 & 71.7 & 59.3 & 47.2 \\
\hline Morocco & 83.9 & 26.3 & 15.9 & 9.7 \\
\hline Oman & 59.4 & 7.3 & 4.8 & 3.2 \\
\hline Palestine & 53.0 & 19.2 & 13.9 & 10.4 \\
\hline Qatar & 23.2 & 6.5 & 4.8 & 3.5 \\
\hline Saudi Arabia & 56.2 & 11.2 & 6.5 & 3.8 \\
\hline Somalia & 127.8 & 79.5 & 59.0 & 41.1 \\
\hline Sudan & 87.0 & 55.0 & 44.8 & 34.3 \\
\hline Syria & 42.9 & 17.7 & 10.0 & 5.5 \\
\hline Tunisia & 65.8 & 15.5 & 9.5 & 6.3 \\
\hline UAE & 29.6 & 5.7 & 3.8 & 2.4 \\
\hline Yemen & 117.0 & 56.2 & 44.4 & 32.0 \\
\hline
\end{tabular}

Source: 1) United Nation, Department of Economic and social Affairs, World Population Prospects: The 2012 Revision, http://esa.un.org/unpd/wpp/unpp/panel_indicators.htm (The Medium Variant).

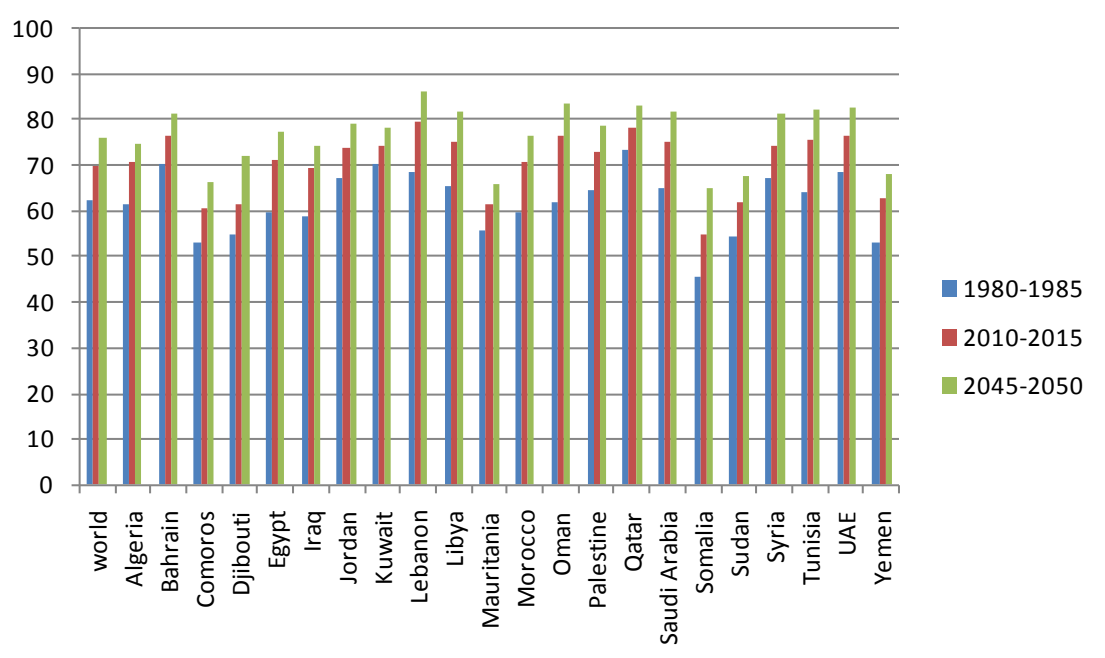

Figure 1. Life expectancy in the world and Arab countries in 1980-2050 (in years). Source: prepared using data from United Nation, Department of Economic and Social Affairs of the United Nations, World Population Prospects: 2012 Revision. 
rates, the Arab region have witnessed profound changes in the age structures of their population, with a rapid increase in the active working group (aged 25 - 64), a decline in the young age group (aged 0 - 14), and a relatively slow but noticeable increase in the number of people aged 65 and older (see Figure 2) 9 (Yount et al., 2009; ESCWA, 2008, 2014).

Compared to more developed regions, the increase in the older population is less substantial in the Arab region, where the population remains relatively young. The percentage of older persons (65 years and above) in the Arab region increased from (3.6 \%) percent in 1980 to (4.1\%) in 2010, and is projected to reach (11.7\%) by 2050 (see Figure 2). The pace of aging however, varies markedly between countries in the region. Countries such as Somalia, Palestine, Comoros and Mauritania will experience the slowest growth in an older population while countries like Lebanon, Tunisia and UAE are most likely to experience a rapid increase in the elderly population (ESCWA, 2013, 2014).Therefore this gradually growing number of older people in the Arab region will put pressure not just on society as a whole but on families' ability to support and care for their older members, as the majority of the older population in the Arab region lives with their families and relies on care provided by their family members.

\section{Family Caregiving for Old Persons in the Arab Region}

It is well established in most Arab countries that the family, as a social institution, is the main care provider for its older adult members. The family is particularly important for older persons, especially when they require assistance due to debilitating chronic conditions and diseases, such as severe arthritis, disability, significant sensory loss, Parkinson's disease, Alzheimer's disease, or when they are otherwise frail [15].

Since Arab culture stresses respect for older people, values highly the natural bond of affection between all members of the family and places enormous obligations on family to support members in old age, most older adults in Arab countries live at home and receive care from one or more family members like children, spouses or other close relatives (Sinunu et al., 2008; Yount et al., 2009). In view of that, the family as a social institution assumes responsibility for enhancing or maintaining older adults' quality of life and their survival and wellbeing by assisting them with the daily chores, including personal care activities (e.g., bathing, eating, dressing, mobility, meal preparation, grocery shopping and making telephone calls), illness-related care (e.g., managing symptoms, coping with illness behaviours, carrying out treatments), care management (accessing resources, communicating with health care professionals, communicating with and navigating the health care and social services systems and acting as an advocate quality care and services), financial management (direct financial assistance and help with bill-paying) and emotional support [16] (Abyad, 2001; Walker, 1995); (Smith, 1994) [17]-[19]. Nevertheless, with industrialization, increased women's participation in the work force and a decline in the extended family there is increasing pressure on families who are responsible for the care of older persons.

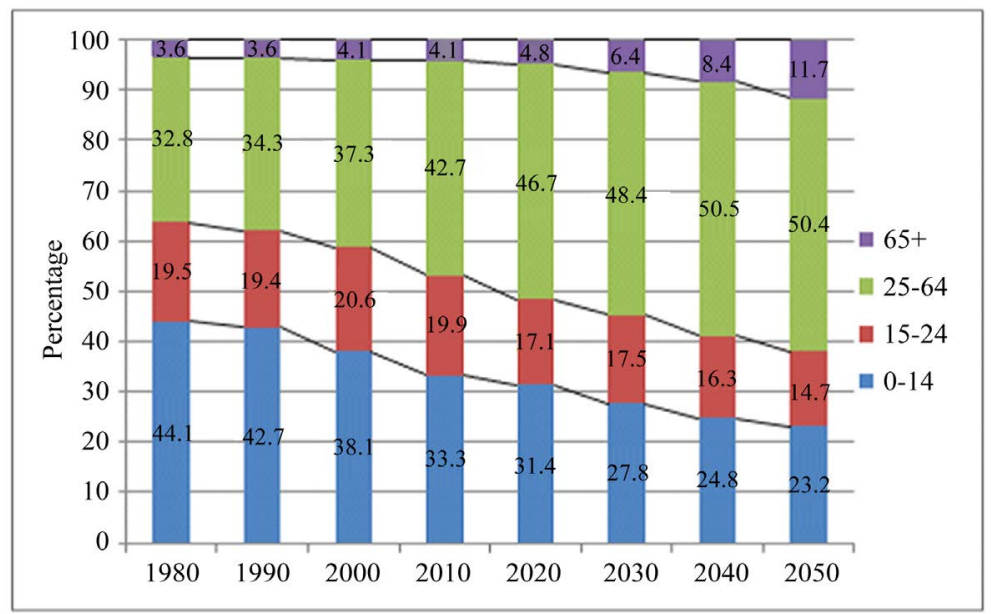

Figure 2. Arabregion population by Broad Age Groups, 1980-2050. Source: Economic and Social Commission for Western Asia (ESCWA) (2013). "The Demographic Profile of Arab Countries”. Technical Paper. 14. United Nations, New York. 
Family caregivers are more likely to experience significant physical, psychological and financial stresses in association with their care-giving role, which “ in turn” can negatively impact their well -being and the quality of life of older persons for whom they provide care. This is particularly pertinent for women who have long been recognized as the primary caregivers for older family members in Arab societies (Sinunu et al., 2008; Yount et al., 2009) [20]-[22].

\section{Challenges Facing Home Caregivers}

Due to the decline in fertility in the Arab world that occurred through the 1980s, several Arab countries are already experiencing population ageing and in the majority of Arab countries it is imminent. Research has proved that there are high levels of chronic, non-communicable diseases and disabilities among the current population of older Arabs and these levels are expected to increase (Yount et al., 2009). It was stated that due to the trends in the demography of ageing in Arab countries this has been accompanied by epidemiological transition with non-communicable diseases as the leading causes of death and morbidity. Studies among older adults have focused on diseases such as musculoskeletal problems, hearing and vision problems and diseases of the circulatory system and their major risk factors such as hypertension, diabetes, obesity, cancer and smoking (Ibid). Most people with these diseases are being cared for by home caregivers, mainly family members or hired non-professional maids. Additionally, the high prevalence of non-communicable diseases and disability among older persons suggests a need for specialized health care for this segment of population. This is because Arab culture places great respect and value on old persons and the obligation on the family to support them (Ibid) (Sinunu et al., 2008). It is clear that most old persons live at home and receive care from their children, spouses or other close relatives.

Several authors have noted that to date, the number of institutions responsible for older persons remains low in most Arab countries (Al-Shammari et al., 2000; Boggatz et al., 2009; Margolis et al., 2001). This is because the dependence on house helpers and full time maids to care for the old person is likely to contribute to lower rates in forming institutions (Yount et al., 2009; Margolis et al., 2001). However, the decline in extended families, the education of women and their increased participation in the labour force made families to institutionalize older adults (Yount et al., 2009; Al-Shammari et al., 2000). Recent reports showed increased numbers of old persons in institutions in the Arab countries, in particular those with the most severe disabilities 2001(Margolis et al., 2001; Sinunu et al 2008).

While an aging population has been a priority in western countries for a long time, it only recently has become a concern in the Arab countries, but there are challenges in many areas facing home caregivers in the Arab region. General issues that Arab countries face with aging are the lack of specialization in teaching of geriatric medicine in all Arab countries. Nursing homes are not well prepared to house chronic patients. Moreover, the older persons are treated by general internists or practitioners and in the GCC are admitted to acute care hospitals. Many older persons complain about inaccessibility of unavailability of specialized home services and lack of social or economic support to the caregivers (Youssef, 2005) [23]-[25].

The next sections will address the main challenges that home caregivers face in taking care of older persons among them are skills and training needed in communication, cultural relevance, abuse of the elderly, and health and medical challenges.

\subsection{Skills and Knowledge}

It has been rightly noted in much research that caregivers do not have the necessary skills and knowledge to provide sustained care for a person with chronic illness or frail older persons, in particular elderly persons who have multiple conditions, such as non-communicable diseases mentioned above, and require treatment protocols. Family caregivers in western societies have commented that they receive little guidance from providers do not know how to assume the caregiver role, are not familiar with the type and amount of care needed, and do not know how to access and utilize resources. This contributes to distress and uncertainty. There is limited research in this area in Arab countries as the field is new and the role of family caregivers has not been investigated to any great extent (Given et al., 2008) [26] [27].

Most of the research done so far explores the emotional health of the caregivers, especially those taking care for people with dementia. The research documents that caring for older persons with dementia does impact the emotional and physical health of the caregiver and little knowledge and information are available for the family 
to provide care or how their knowledge and skills affect care (Given et al., 2008) [28]-[30]. It was noted, however, that most studies do not organize or classify interventions according to caregivers' tasks or the knowledge and skills they require. This information is vital in the planning and implementation of the interventions that will help them. That is why recently researchers have suggested that family caregivers require both knowledge and skill to provide the necessary care and to reduce their own distress (Given et al., 2008) [31] [32], Schumacher et al. 2000.

Schumacher and others (2000) defined family care-giving skills as "the ability to engage effectively and smoothly in nine care processes requiring psychomotor, cognitive and psychological skills." Skills were defined as "goal directed, well-organized behavior that is acquired through practice and performed with an economy of effort" (Schumacher et al., 2000). Additionally, Farran defined caregiver skill as something that caregivers do using goal-directed behaviours based on knowledge, experience or personality style (Farran et al., 2007; Given et al., 2008). The basic knowledge that caregivers use to make decisions and solve problems provides the foundation for developing and improving skills. Caregivers should receive guidance, counselling and direction from health clinicians and rehearse what they learn in order to take appropriate action with their family members. Over time, caregivers can develop their skills (ibid). The skills to which Schamacher refers include monitoring, interpreting, making decisions, taking actions, adjusting to changing needs, comforting with hands-on care, accessing resources, working with the sick persons and negotiating the health care system (Schumacher et al., 2000; Given et al., 2008).

Furthermore, the demands for providing care depend on many factors which vary with the patient's personality, the type and stage of illness, the caregiver's physical, cognitive, social, organizational and psychological knowledge and skills. Therefore, it is important to assess the care demands while evaluating the caregiver's availability, capacity, knowledge, skills, and competing family roles and resources because it is vital to consider the routine nature of the care as well as the duration and quantity in terms of hours of caring when implementing plans for caring of the family (Given et al., 2008). This issue is not studied sufficiently and there is very little research in the Arab region into the demands on caregivers.

In addition, although in this paper we are not specifically targeting specific illness or sickness or a particular age group of caregivers, in general caregivers need to perform complex medical tasks, supervise the older person, make decisions, solve problems, provide emotional support, and coordinate care. Using these skills, caregivers administer medications, plan and provide meals, handle medical equipment, and provide direct care such as wound care and lifting and turning. As caregivers they also provide custodial care, transportation, and advocacy. All these tasks are time consuming in addition to managing the household. However, there is very scarce information and research in the Arab countries available on how family caregivers fulfil these tasks and provide the skills and knowledge needed for them to perform the task. Caregivers also need to modify the environment in which the older person is staying by acquiring equipment or assistive devices to improve the functions and safety of the older person. These are critical issues that we need to look at when considering the care of the elderly. In fact the demand increases as the treatment changes or the disease progresses or the older person's functional or cognitive capacity deteriorates. Those issues need to be taken into consideration and the role of professional nurses or doctors is needed to reassess the capabilities of the caregivers as to whether they can handle the situation.

Given et al. demonstrate that not only that caregivers do need skills and knowledge, and the required knowledge and skills are beginning to be recognized and identified, but researchers have paid little attention to interventions by health clinicians. It is true that most interventions provide basic written materials that inform caregivers about the expected course of illness, treatment plans etc. but family caregivers need a working knowledge of the patient's plan of care and must be able to interact with nurses and social workers.

Skills are based on knowledge and can be classified as psychomotor, such as wound care, cognitive, such as monitoring, decision making and problem solving, or psychological, such as offering emotional support, and most importantly, the ability to communicate effectively, which will be discussed later in this paper. However, few studies in the Arab region look at how care is given and what care family caregivers should provide for the elderly.

Given that the necessary knowledge and skills vary depending on the patient's illness and type, researchers should look more at evaluating caregivers' knowledge and skills and capacity. Assessments should include the number of hours care required; the expected duration of care; and take into consideration the demands of work, travel, and child care, which can increase the risk of overload, strain or inability to continue to provide care 
(Given et al., 2008).

Overall, health care providers should cooperate with the family caregivers, supporting them, and providing them with the appropriate knowledge and skills in order to achieve a high quality of care. Health care providers also should help family caregivers develop problem solving and organizational and communication skills. It is also important that health professional need to help family members acquire skills while keeping in mind their cultural and ethnic differences. Formal interventions from the health providers are needed to achieve optimal clinical outcomes.

\subsection{Communication Strategies}

One of the important challenges that family caregivers face is communication. Corcoran defined communication as "a complex interaction that requires adequate production, transmission and reception of information, many factors can work to impede communication and lead to frustration, wasted effort, resistance to care or an unsafe situation" [33].

It is widely accepted that "communication involves sending, receiving, understanding and utilizing information. Attitudes, feelings, and behaviors can cause breakdowns in communication between people of different generations. Miscommunication can cause people to respond or react negatively.” [34].

Studies have paid much attention to communication and identified three elements for effective communication-acknowledgement, participation, and engagement. It is important to realize that this also applies to older persons. Sadly, however, many older people are overlooked and not always acknowledged, and are the objects of condescension. Participation means having a part or share and being aware of the issues that could affect a person's ability to participate in a conversation. Language and cultural background can be a challenge. Finally, engagement literally means to interest and to involve. Family caregivers need to speak in a way that encourage involvement and engage the older person in conversation and to be aware of their tone and choosing words carefully. Older persons could feel embarrassed, threatened or offended and as a result they will shut down (University of Cincinnati College of Nursing, 2011).

From the above definitions and explanations, we can classify t communication in two categories: verbal and non-verbal. Verbal communication is defined as the timing and content of spoken language. It is best to keep the message very simple and use the fewest words possible to get the information across. ${ }^{\cdots}$ Difficulty with communication can be very frustrating for both caregiver and care recipient, possibly leading to a catastrophic reaction (Corcoran, 2003). Another definition of verbal communication style is that:

Verbal communication includes cultural difference in vocabulary, grammatical structure, voice qualities, intonation, rhythm, speed, pronunciation, and silence. One example of something that is very divisive to communication in the Arab world is the prevalence of dialects. Dialects of the Arabic language not only vary by region, but vary by country or even from city to city or village to village [35].

Initiating verbal communication is influenced by cultural values. For example, it is important for the home caregiver to take into consideration the need to take ample time to listen to the older person and answer questions and discuss topics; to avoid slang words, medical terminology, and abbreviations; to use augment spoken conversation with gestures or pictures in order to increase the client's understanding; to speak slowly in a respectful manner and at a normal volume; to frequently validate the old person understanding of what is being communicated; and to not automatically interpret a client's smiling and nodding to mean that the older person understands, this might not be the case (Kozier, 2011). These issues are rarely discussed or implemented effectively in most cases with family home caregivers. They need more research and empirical data on how home caregivers communicate adequately with their older family members, in particular in Arab countries where more than one family member takes care of the elderly.

For communication to be effective, the home caregivers need to be aware of age related issues, such as hearing impairment, deteriorating sight, poor memory, and the inability to read or understand. Other issues might include the type of information being shared, understanding of an issue or topic, and the environment and personal style such as the use of body language, tone of voice, words chosen, speaking etc (University of Cincinnati College of Nursing, 2011).

In communicating with older persons it is also important to notice that each has their own personality. Some are quiet and others are loud and assertive etc. Moreover, it depends on their condition and what disease they have. For example, communicating with an old person who has dementia is different from one with impaired 
hearing or with Alzheimer. The family caregivers need to know the skills needed in communicating with an older person, and research has shown that there are many guidelines and tools of communication between the caregiver and the elderly person. Yet this is beyond the scope of this paper (see family caregiver online 2004, elder care online, My Dr, 2001).

Non-verbal communication can include the use of silence, touch, eye movement, facial expressions, and body posture. There are some cultures that are comfortable with silence whereas others consider it appropriate to speak. Touching can involve learned behaviours that can have both negative and positive meaning. In some cultures, it is not acceptable to be touched while in others shaking hands is a meaningful and conveys character and strength. Facial expression can also vary between cultures. People from some cultures are more likely to smile and use facial expression to communicate feelings. Eye movement during communication has cultural foundations. For example, in western societies direct eye contact is regarded as important and shows that the other is attentive and listening. On the other hand, other cultures may view eye contact as impolite or invasion of privacy (Kozier et al., 2011). Moreover, body posture and the hand-shake are also learned according to culture and those are all points that need to be considered carefully when family caregivers communicate with their older members (Ibid) because it affects the quality of life of both the caregiver and the older person. Generally, most home caregivers face obstacles in taking care of their elderly relatives because they lack the right communication skills, leading to the burden of health issues that have an impact on the health of the older person. These issues are rarely studied in the Arab countries.

\subsection{Cultural Relevance}

It is important for family caregivers to consider the culture in which they are living and that of the older person when taking care of their elderly relatives. Sometimes there is more than one family member taking care of an elderly person while in some societies a number of families request the help of other members of their extended family in supplying care. Here the family caregiver needs to take into consideration the culture in which the older person was living, in particular since there can be gaps between the generations and we need to respect the sensitivity of the culture. Thus, to give a level of professional care to their older members, family caregivers should know that nursing is culturally sensitive and should be culturally appropriate and competent. It is a proviso to take into consideration the cultural boundaries of the older person, as well as the situations in which the person's health problem has arisen.

Kozier et al. (2011) defined "culturally sensitive as implying that nurses possess some basic knowledge of a constructive attitudes toward the health traditions observed among the diverse cultural groups found in the setting in which they are practicing.

Culturally appropriate implies that nurses (caregivers) apply the underlying background knowledge that must be possessed to provide a given client with the best possible health care.

Culturally competent implies that, in the care delivered, caregivers understand and attend to the total context of the older person's situation and use a complex combination of knowledge, attitude and skills” (Ibid).

It has been rightly remarked that people face issues in adapting to their environment in matters such as providing nutrition, shelter, caring for and educating children, dividing labour, developing social organization, controlling disease and health etc. Culture "refers to norms and practices of a particular group that are learned and shared and guide thinking, decision and actions. Culture is a universal experience but no two cultures are exactly alike. Cultural patterns are learned and it is important for nurses (caregivers) to note that members of a particular group may not share identical cultural experiences. Each member of a cultural group will be somewhat different from his or her cultural counterparts. For example, third generation Arabs will differ in their cultural understanding from first generation Arabs” (Ibid).

Overall, it is important to establish a mutual relationship between the caregiver and the elderly person. That is to say, a cultural sensitive environment is needed and this involves a process of how and when questions are asked. Sensitivity is important in the timing and phrasing of questions since this need to be adapted to the individual.

\subsection{Abuse of the Elderly}

Elder abuse is not a new phenomenon. It is a problem that exists in many countries around the world yet is globally underreported. While there is little information regarding the extent of abuse among elderly populations, 
especially in developing countries, it is estimated 1 in 10 older people experience abuse each month. In fact, elder abuse is not limited to any one gender, religious, cultural, ethnic or income group may occur in many different settings, including private homes, residential care settings, and hospitals. However, data on the extent of the problem in such settings, specifically in developing countries are scarce (Corcoran, 2003) [36] [37]. According to the World Health Organization's data (2015), as many countries around the world have rapidly aging population, the number of cases of elder abuse is expected to increase. It is predicted that by the year 2050, the global population of people aged 60 years and older will more than double, from 900 million in 2015 to about 2 billion.

Elder abuse is a term "referring to any knowing, intentional, or negligent act by a caregiver or any other person that cause harm or a serious of risk of harm to a vulnerable adult” [38]. The term elder abuse and neglect is also used to describe acts of commission or omission that result in harm or threatened harm to the health or welfare of an older adult. This abuse or mistreatment can be intentional or unintentional. Intentional mistreatment involves "a conscious or deliberate attempt to inflict harm or injury, such as verbal abuse or battering; unintentional mistreatment occurs when an inadvertent action results in harm to elderly persons. Unintentionally is done due to ignorance, inexperience or lack of ability or desire of the caretaker to provide proper care” [39]. There are different types of elder abuse that can be categorized into self-abuse, domestic elder abuse, and institutional elder abuse. Self-abuse or self-neglect is the result of the older persons unwillingness to look after themselves as a result of depression from the loss of a spouse, loneliness, chronic pain, financial worry and loss of interdependence.

Domestic and institutional abuse can be divided into different sub-categories -physical abuse, emotional abuse, sexual abuse, exploitation, neglect and abandonment. However, it is difficult to detect victims of abuse and both older men and women are at risk. In fact elder abuse is not reported, it is hidden and most elderly abused victims do not report the incidences due to fear of being punished, fear of institutionalization, fear of rejection from family members, fear of losing their caregiver, shame or guilt, lack of understanding or impairment, unaware of resource options etc (Latha, 2008). Research in this area is limited, particularly in Arab countries. This phenomenon of elderly abused so far has been studied only in Lebanon, Israel, Yemen, and Cairo. The study conducted in Yemen revealed the various types of elder abuse and it showed that psychological abuse had the highest prevalence whereas physical abuse was the lowest [40]. The study in Cairo concluded that providing care for an elder person is stressful and how much burden can cause abuse to the elderly. The studies recommended new interventions for better in-home services that better meets the social needs of old people [42] (Sinunu et al., 2008). Other Arab countries have very limited information on the issue. Therefore, it is important for caregivers to know how to deal with the situation when an elderly person is abused and to overcome this problem and find strategies to remedy the situation. According to Gupta et al. [41], a large proportion of elder abuse takes place in shared living arrangement. Those who live alone are more exposed to financial abuse and elderly patients with dementia are at risk of physical abuse.

Research has given explanations for the characteristics of the elderly abused person and the caregiver. In majority of cases the abused person is female, widowed, having a low income and low educational level. In addition to dependency as a result of disability, limitations in the activities of daily life and dementia increase the risk of abuse. The main characteristics of the caregiver have been revealed to be stress and burn out and frustration leading to abuse of their ward. Poor anger management, alcoholism, financial constraints, and financial burden are additional causes. The personality and psychological character of the caregiver as well as that of the patient can play a causal role in situations involving mistreatment (Gupta, 2008; Walker et al., 1995).

\subsection{Health and Medical Issues}

Family home caregivers are greatly exposed to health and medical issues that can have an impact on taking care of their elderly relatives. Walker et al. (1995) indicated that caring for an elderly relative can result in problems such as care-giving burden, stress and depression. The burden of caregiving refers to the physical, psychological, and social impact that caring for older persons has on the caregiver. Stress is a common symptom of many caregivers taking care of older people and those who experience stress are more fatigued and could experience adverse health impacts. Studies have shown that the level of stress varies from one caregiver to the other. For example, in a study done by Ahmad [42] revealed that working caregivers were more stressed than those of nonworking caregivers because they had to take some time off from their work to take care of their elderly relative 
and this caused them stress and fatigue; and caregiver distress is greater when the older person symptoms are severe (Walker et al., 1995). When taking gender into consideration caregiving is more stressful for women than men. Also, caring for a cognitively impaired family member is more stressful than caring for a physical impaired older person (Ibid). In addition, the most significant factors with caregivers who need to give a greater degree of care and responsibility are the impact that this has on the caregiver's schedule and health. Furthermore, it has been reported that depression in the elderly is associated with depression in their caregivers (Ibid).

Studies showed that as the world's population is growing older and people tend to take the responsibility of caring for their older family members in their own home, they are exposed to stressful situations that might increases their risk of developing several physical afflictions as well as emotional problems, such as anxiety and depression. Nevertheless, interventions have been designed to assist the caregivers through community services, like day centres, home care services, educational programmes in which information is given about the older person illness and the skills necessary to deal with care-related problems, mutual help groups that provide caregivers with support and someone to listen, and psychotherapeutic interventions [43]. In the Arab countries, however, these health issues that affect caregivers do not receive that much attention. Only limited research has been done on the fact that caregivers are vulnerable to stress and health issues due to their care-giving role and the interventions to remedy these (Zahid et al., 2010) [44]. In fact, few studies have considered the importance of the burden on caregivers, but among them a study done in Iran that showed that the family burden led to important issues in health and impacts on the life of both the elderly person and the caregiver and describes the strategies to overcome this (Ibid).

\subsection{Financial Burden}

Family caregiving can be costly but there is limited empirical research on the effects of financial costs on family caregiving. As mentioned previously, caregiving affects a caregiver's work and family financials such as providing care to a family member and working full time. Financial cost refers to "direct momentary costs in employment due to one having to perform a caregiving role. Not all financial costs could be measured by momentary values as some cost is subjectively indicated by individuals as perceived financial costs” [45].

Family caregiving is affected by the daily costs of caregiving. Such as caregiving expenses related to transportation, non-prescription medication, medical supplies, prescription medications, equipment, home making supplies can affect the financial budget of the family. This is because most of these items are not covered by insurance programs (Lai, 2012).

The situation is worse in Arab countries as there are poor countries such as Sudan, Djibouti that do not have an insurance program for the old people. Most of the old people are taken care by their family members and this cost financial burden. The impact of caregiving on financial cost in Arab countries has not been studied and there is no empirial data to explain the effect. Thus more research is needed in this area.

\section{Conclusion and Policy Implications}

This paper has shown the importance of research in the Arab countries for elderly people and how limited research there is currently. The concept and idea of ageing is emerging in the Arab countries as data has shown there is a projected increase in ageing populations by 2050 and attention is needed for the question of who will take care of the elderly people. Thus, it is important to conduct research not only on elderly people and their carers but also on the facilities, services and support available to them from the state. By doing so an agenda can be constructed giving priority to the needs of the elderly and those involved in activities and programmes related to them. In fact, the construction of programmes will benefit the lives of the older person and provide them with healthy lifestyle and a better quality to life.

It is important to highlight in this section that research is needed for the home caregivers in the Arab countries. More research must be conducted on home care services and the role of research in studying the impact, the advantages and disadvantages of home care in the Arab region. A new phenomenon in research/data in Arab countries studying cultural relevance, elder abuse and quality of life, but this is limited as is the research into the interrelation amongcultural relevance, abuse and quality of life. The involvement of older people in research relating to them is important in sharing their experience and knowledge, increasing the acceptability and accessibility of conducting research by the community.

In addition, there are policy implications that would support the research and benefit home caregivers in the 
important roles they are performing. Based on the literature discussed in this paper, we recommend prioritization of a comprehensive agenda for older people because the current agenda is limited and insufficient to meet the growing elderly population needs. The concerns of the elderly themselves should be noted and should be reflected in this agenda. The social policy and financial strategies are needed to address this issue and the demands on the workforce who will provide the care: in addition to promoting a healthy lifestyle to provide effective services of health care and long-term care by supporting social integration including strengthening diverse family types, extending social ties beyond the family and giving aid to caring families is also needed, moreover, it should encourage societal participation of the community by: a) reinforcing employability and stimulating employers and b) creating opportunities for volunteering. Families taking care of their elderly need to be supported and educated to help them understand their role and the needs of the elder whom they care. An improved knowledge base approach on elder violence and abuse is needed to develop and implement strategies and programmes for intervention.

\section{Disclaimer}

The paper reflects the personal opinion of the authors and is not necessarily endorsed by the Doha International Family Institute.

\section{References}

[1] Palloni, A., Peláez, M. and Wong, R. (2006) Introduction: Aging among Latin American and Caribbean Populations. Journal of Aging and Health, 18, 149-156. http://dx.doi.org/10.1177/0898264306286766

[2] Economic and Social Commission for Western Asia (ESCWA) (2014) Aging in the Arab Region: Trends, Implications and Policy Options. E/ESCWA/SDD/2013/Technical Paper. 15, Beirut.

http://www.escwa.un.org/divisions/div editor/Download.asp?table name=divisions other\&field name=ID\&FileID=1 $\underline{588}$

[3] Abdulrahim, S., Ajrouch, K.J., Jammal, A. and Antonucci, T.C. (2012) Survey Methods and Aging research in an Arab Sociocultural Context—A Case Study from Beirut, Lebanon. Journal of Gerontology, 67, 775-782. http://dx.doi.org/10.1093/geronb/gbs083

[4] Yount, K.M. and Sibai, A.M. (2009) Demography of Ageing in Arab Countries. In: Uhlenberg, P., Ed., International Handbook of Population Ageing, 1, Springer, Netherlands, 277-315.

[5] Sinunu, M.A., Yount, K.M. and El-Afify, N.A. (2008) Informal and Formal Long-Term Care for Frail Older Adults in Cairo, Egypt: Family Caregiving Decisions in a Context of Social Change. Journal of Cross Cultural Gerontology, 24, 63-76. http://dx.doi.org/10.1007/s10823-008-9074-6

[6] Economic and Social Commission for Western Asia (ESCWA) (2008) Situational Analysis of Population Aging in the Arab Countries: The Way Forward Towards Implementation of MIPAA. E/ESCWA/SDD/2008/ Technical Paper. 2, United Nations, New York. http://www.globalaging.org/elderrights/world/2008/situation.pdf

[7] Given, B., Sherwoon, P. and Given, C. (2008) What Knowledge and Skills Do Caregivers Need? Journal of Social Work Education, 108, 115-123. http://dx.doi.org/10.5175/JSWE.2008.773247703

[8] United Hospital Fund (2014) Home Care: A Family Caregiver Guide. http://www.nextstepincare.org/uploads/File/Guides/Home Care/Guide/Home Care.pdf

[9] Al-Shammari, S.A., Al Mazrou, Y., Jarahhal, J.S., Al Ansary, L., El-Shabrawy, A.M. and Bamgboye, E.A. (2000) Appraisal of Clinical, Psychological and Environmental Health of the Elderly in Saudi Arabia: A Household Survey. International Aging Human Development, 50, 43-60. http://dx.doi.org/10.2190/17YQ-9R9E-YG86-HYN1

[10] Abyad, A. (2001) Health Care for Older Persons: A Country Profile-Lebanon. Journal of American Geriatrics Society, 49, 1366-1370. http://dx.doi.org/10.1046/j.1532-5415.2001.49268.x

[11] Precision Home Health Care (2014) Benefits of Home Care for the Elderly. http://www.precision-hhc.com/benefits-of-home-care-for-the-elderly/

[12] Saxena, P. (2008) Ageing and Age-Structural Transition in the Arab Countries: Regional Variations, Socioeconomic Consequences and Social Security. Genus, 64, 37-74.

[13] United Nations (2012) World Population Prospects: The 2012 Revision. Department of Economic and Social Affairs, United Nations, New York. http://esa.un.org/unpd/wpp/index.htm

[14] Economic and Social Commission for Western Asia (ESCWA) (2013) The Demographic Profile of the Arab Countries. (E/ESCWA/SDD/2013/Technical Paper 14). Beirut. http://www.escwa.un.org/information/pubaction.asp?PubID=1568 
[15] Walker, A., Pratt, C.C. and Eddy, L. (1995) Informal Caregiving to Aging Family Members: A Critical Review. Family Relations, 44, 404-411. http://dx.doi.org/10.2307/584996

[16] National Association of Social of Social Workers (2010) NASW Standards for Social Worker Practice with Family Caregivers of Older Adults. http://www.socialworkers.org/practice/standards/naswfamilycaregiverstandards.pdf

[17] Smith, C.E. (1994) A Model of Caregiving Effectiveness for Technologically Dependent Adults Residing at Home. Advances in Nursing Science, 17, 27-40. http://dx.doi.org/10.1097/00012272-199412000-00006

[18] Schumacher, K.L., Stewart, B.J., Archbold, P.G., Dodd, M.J. and Dibble, S.L. (2000) Family Caregiving Skill: Development of the Concept. Research in Nursing \& Health, 23, 191-203. http://dx.doi.org/10.1002/1098-240X(200006)23:3<191::AID-NUR3>3.0.CO;2-B

[19] Bowers, B.J. (1987) Intergenerational Caregiving: Adult Caregivers and Their Aging Parents. Advances in Nursing Science, 9, 20-31. http://dx.doi.org/10.1097/00012272-198701000-00006

[20] Boggatz, T., Farid, T., Mohammedin, A. and Dassen, T. (2009) Attitudes of Older Egyptians towards Nursing Care at Home: A Qualitative Study. Journal of Cross Cultural Gerontology, 24, 33-47. http://dx.doi.org/10.1007/s10823-008-9069-3

[21] Lowenstein, A. and Katz, R. (2009) Rural Arab Families Coping with Caregiving. Marriage and Family Review, 30, 179-197. http://dx.doi.org/10.1300/J002v30n01_11

[22] Margolis, S.A. and Reed, R.L. (2001) Institutionalizing Older Adults in a Health District in the United Arab Emirates: Health Status and Utilization Rate. Gerontology, 47, 161-167. http://dx.doi.org/10.1159/000052791

[23] Atallah, R., Nehme, C., Seoud, J., Yertzian, J., Zablit, C., Levesque, L. and Ducharme, F. (2005) Caregivers of Elderly People with Loss of Autonomy in Lebanon: What Is the Context of Their Health Care? Recherche en Soins Infirmiers, 81, 122-138.

[24] Keller, I., Makpiaa, A., Kalensher, T. and Kalache, A. (2002) Global Survey on Geriatrics in the Medical Curriculum. World Health Organization, Geneva. http://www.who.int/ageing/publications/alc_tegeme_survey.pdf

[25] Hafez, G., Bagchi, K. and Mahaini, R. (2000) Caring for the Elderly: A Report on the Status of Care for the Elderly in the Eastern Mediterranean Region. Eastern Mediterranean Health Journal, 6, 636-643.

[26] Given, B.A., Given, C.W. and Kozachik, S. (2001) Family Support in Advanced Cancer. CA: A Cancer Journal for Clinicians, 51, 213-231. http://dx.doi.org/10.3322/canjclin.51.4.213

[27] Northouse, L.L., Mood, D., Templin, T., Mellon, S. and George, T. (2000) Couples’ Patterns of Adjustment to Colon Cancer. Social Science Medicine, 50, 271-284. http://dx.doi.org/10.1016/S0277-9536(99)00281-6

[28] Cameron, J.L., Franche, R.-L., Cheung, A.M. and Stewart, D.E. (2002) Lifestyle Interference and Emotional Distress in Family Caregivers of Advanced Cancer Patients. Cancer, 94, 521-527. http://dx.doi.org/10.1002/cncr.10212

[29] Gitlin, L.N., Hauck, W.W., Dennis, M.P. and Winter, L. (2005) Maintenance of Effects of the Home Environmental Skill Building Program for Family Caregivers and Individuals with Alzheimer's Disease and Related Disorders. The Journals of Gerontology: Series A, 60, 368-374. http://dx.doi.org/10.1093/gerona/60.3.368

[30] Pinquart, M. and Sorensen, S. (2003) Associations of Stressors and Uplifts of Caregiving with Caregiver Burden and Depressive Mood: A Meta-Analysis. The Journals of Gerontology: Series B, 58, 112-128. http://dx.doi.org/10.1093/geronb/58.2.P112

[31] Farran, C.J., Gilley, D.W., McCann, J.J., Bienias, J.L., Lindeman, D.A. and Evans, D.A. (2007) Efficacy of Behavioral Interventions for Dementia Caregivers. Western Journal of Nursing Research, 29, 944-960. http://dx.doi.org/10.1177/0193945907303084

[32] Bakas, T., Austin, J.K., Jessup, S.L., Williams, L.S. and Oberst, M.T. (2004) Time and Difficulty of Tasks Provided by Family Caregivers of Stroke Survivors. Journal of Neuroscience Nursing, 36, 95-106. http://dx.doi.org/10.1097/01376517-200404000-00007

[33] Corcoran, M. (2003) Practical Skills Training for Family Caregivers. https://caregiver.org/practical-skills-training-family-caregivers

[34] University of Cincinnati College of Nursing (2011) Aging and Communications: Engaging Older People. Centre for Aging with Dignity. http://nursing.uc.edu/content/dam/nursing/docs/CFAWD/LookCloserSeeMe/Module\%204_GDST_Reference\%20Guid e.pdf

[35] Kozier, B., Harvey, S. and Morgan-Samuel, H. (2011) Fundamentals of Nursing: Concepts, Process and Practice. http://wps.prenhall.com/chet kozier fundamentals $7 /$

[36] World Health Organization (2015) Elder Abuse. http://www.who.int/mediacentre/factsheets/fs357/en/

[37] Glasgow, K. and Fanslow, J.L. (2007) Family Violence Intervention Guidelines: Elder Abuse and Neglect. Ministry of Health, Wellington. http://www.moh.govt.nz 
[38] Gupta, R. and Chaudhuri, A. (2008) Elder Abuse in a Cross-Cultural Context: Assessment, Policy and Practice. Indian Journal of Gerontology, Special Issue on Elder Abuse and Neglect, 22, 373-393.

[39] Latha, K. (2008) Elder Abuse and Neglect: A Review. Indian Journal of Gerontology, Special Issue on Elder Abuse and Neglect, 22, 467-479.

[40] Ibrahim, H. and ElSelwy, K. (2006) Elderly Abuse in El Hodeidah City, Yemen. El-Minia Medical Bulletin, 17, 245-257.

[41] Salama, R. and El-Soud, F. (2012) Caregiver Burden from Caring for Impaired Elderly: A Cross Sectional Study in Rural Lower Egypt. Italian Journal of Public Health, 9.

[42] Ahmad, K. (2012) Informal Cregivers to Chronically III Older Family Members: Caregiver’s Experiences and Problems. South Asian Studies. A Research Journal of South Asian Studies, 27, 101-120.

[43] Lopez, J., Crespo, M. and Zarit, S. (2007) Assessment of the Efficacy of a Stress Management Program for Informal Caregivers of Dependent Older Adults. The Gerontologist, 47, 205-214. http://dx.doi.org/10.1093/geront/47.2.205

[44] Zahid, M. and Ohaeri, J. (2010) Relationship of Family Caregiver Burden with Quality of Care and Psyopathology in a Sample of Arab Subject with Schizophrenia. BMC Psychiatry, 10, 71. http://dx.doi.org/10.1186/1471-244X-10-71 http://www.ncbi.nlm.nih.gov/pmc/articles/PMC2945972/

[45] Lai, D. (2012) Effect of Financial Costs on Caregiving Burden of Family Caregivers of Older Adults. Sage Open, 2. http://dx.doi.org/10.1177/2158244012470467 